Tail behavior of the generalized Maxwell distribution

Huang, Jianwen and Chen, Shouquan and Tuo, Zhongyou

2014

MIMS EPrint: 2014.17

Manchester Institute for Mathematical Sciences

School of Mathematics

The University of Manchester

\footnotetext{
Reports available from: http://eprints.maths.manchester.ac.uk/

And by contacting: The MIMS Secretary

School of Mathematics

The University of Manchester

Manchester, M13 9PL, UK
} 


\title{
Tail behavior of the generalized Maxwell distribution ${ }^{*}$
}

\author{
${ }^{a}$ Jianwen Huang $\quad{ }^{b}$ Shouquan Chen $\quad{ }^{a}$ Zhongyou Tuo \\ ${ }^{a}$ School of Mathematics and Computational Science, Zunyi Normal College, Zunyi, 563002, China \\ ${ }^{b}$ School of Mathematics and Statistics, Southwest University, Chongqing, 400715, China
}

\begin{abstract}
In this paper, we investigate the tail properties of the generalized Maxwell distribution and gain an asymptotic behavior of Mills-type ratio. Meanwhile, We show two applications. The first application thinks about the asymptotic property of the ratio of density functions and the ratio of the tails of the generalized Maxwell and classical Maxwell distributions. Another application obtains the asymptotic distribution of the partial maximum of an independent and identically distributed sequence from the distribution.
\end{abstract}

Keywords. Extreme value distribution; Generalized Maxwell distribution; Maximum; Mills-type ratio.

Mathematics Subject Classification(2010) Primary 62E20, 60E05; Secondary 60F15, 60G15.

\section{Introduction}

Generalized Maxwell distribution was introduced by Vodă (2009). The distribution has a variety of areas that range from statistics to physics, particularly in statistical mechanics. Some recent examples of this are: constructing fractional rheological constitutive equations (Schiessel et al., 1995); be friction model suitable for quick simulation and control (Farid et al., 2005); forecasting the temporal change of opening angle in multiple time scales and electroscalar wave (Zhang et al., 2008; Arbab and Satti, 2009); project of the time related to behavior of viscoelastic materials (Monsia, 2011). The properties of it also have been studied in some literature, see Liu and Fu (2013) and Liu and Liu (2013). The probability density function (pdf) of the generalized Maxwell distribution (denote by GMD) is given by:

$$
g_{k}(x)=\frac{k}{2^{k / 2} a^{2+1 / k} \Gamma(1+k / 2)} x^{2 k} \exp \left(-\frac{x^{2 k}}{2 a^{2}}\right)
$$

for $a, k>0$ and $x>0$, where $\Gamma(\cdot)$ denotes the Gamma function. When $k=1$, the distribution of density function (1.1) can derive the classical Maxwell (or called ordinary Maxwell) pdf, that is,

$$
g_{1}(x)=\sqrt{\frac{2}{\pi}} \frac{x^{2}}{a^{3}} \exp \left(-\frac{x^{2}}{2 a^{2}}\right) .
$$

* This work was partially supported by the NSFC (grant No. 11071199, and No. 11171275) and the SWU Grant for Statistics Ph.D.

${ }^{\dagger}$ Corresponding author. E-mail address: hjw1303987297@126.com 
Throughout the paper, notation $G_{1}(x)$ represents the cumulative distribution function (cdf) of the classical Maxwell distribution (for short MD).

Mills (1926) gave a well-known inequality and Mill's ratio for the standard normal cdf $\Phi(x)$ with pdf $\phi(x)$ as follows:

$$
x^{-1}\left(1+x^{-2}\right)^{-1} \phi(x)<\Phi(-x)<x^{-1} \phi(x), \text { for } x>0,
$$

and

$$
\frac{\Phi(-x)}{\phi(x)} \sim \frac{1}{x}, \text { as } x \rightarrow \infty
$$

Peng et al. (2009) extended the above results to the case of the general error distribution. Lin and Peng (2010) derived the similar results of short-tailed symmetric distribution. Lin and Jiang (2012) considered a generalization of the short-tailed symmetric distribution and the asymptotic behavior of Mills-type ratio about the distribution family.

The inequalities like (1.2) and (1.3) are really important in considering some tail behavior of economic and financial data. However, to our knowledge, there is no study concerning GMD. In this paper, we derive the above-mentioned inequality, Mills-type ratio and the tail distribution representation of GMD. Two applications are provided. One application investigates the asymptotic behaviors of the ratio of the pdfs and the ratio of the tails of the GMD and the classic Maxwell distribution. Another important application obtains the limiting distribution of the maximum of independent and identical GMD random variables.

The rest of the paper is organized as follows: In Section 2, we present an inequality and Millstype ratio similar to (1.2) and (1.3) for the GMD. In Section 3 and 4, we show applications of the results. In Section 3, we investigate asymptotic properties of the ratio of the pdfs and the ratio of the tails of the GMD and the MD. In Section 4, we consider the limiting distribution of the partial maximum of an independent and identically sequence from the GMD. Here, our work is to choose the suitable normalizing constants such that the distribution of the maxima belongs to the domain of attraction of $D(\Lambda)$, where $\Lambda(x)=\exp (-\exp (-x))$.

\section{Mills Ratio of GMD}

The following inequality is crucial to our result.

Lemma 2.1. Set $k>\frac{1}{2}$. For all $x>0$, we have

$$
\int_{x}^{\infty} \exp \left(-\frac{t^{2 k}}{2 a^{2}}\right) \mathrm{d} t<\left(\frac{k x^{2 k-1}}{a^{2}}-\frac{1}{x}\right)^{-1} \exp \left(-\frac{x^{2 k}}{2 a^{2}}\right)
$$

Proof. For $x>0$,

$$
\begin{aligned}
x^{2 k} \int_{x}^{\infty} \exp \left(-\frac{t^{2 k}}{2 a^{2}}\right) \mathrm{d} t & <\int_{x}^{\infty} t^{2 k} \exp \left(-\frac{t^{2 k}}{2 a^{2}}\right) \mathrm{d} t \\
& =\frac{a^{2} x}{k} \exp \left(-\frac{x^{2 k}}{2 a^{2}}\right)+\frac{a^{2}}{k} \int_{x}^{\infty} \exp \left(-\frac{t^{2 k}}{2 a^{2}}\right) \mathrm{d} t .
\end{aligned}
$$


Hence,

$$
\int_{x}^{\infty} \exp \left(-\frac{t^{2 k}}{2 a^{2}}\right) \mathrm{d} t<\left(\frac{k x^{2 k-1}}{a^{2}}-\frac{1}{x}\right)^{-1} \exp \left(-\frac{x^{2 k}}{2 a^{2}}\right)
$$

The following results consist of an inequality and Mills-type ratio which are similar to (1.2) and (1.3), respectively.

Lemma 2.2. Set $k>\frac{1}{2}$. For all $x>0$, we have

$$
\frac{a^{2}}{k} x^{1-2 k}<\frac{G_{k}(-x)}{g_{k}(x)}<\frac{a^{2}}{k} x^{1-2 k}\left(1+\left(\frac{a^{2}}{k} x^{2 k}-1\right)^{-1}\right),
$$

where a is positive.

Proof. Observe the symmetry of the GMD. For $x>0$, we have

$$
\begin{aligned}
G_{k}(-x) & =\int_{x}^{\infty} g_{k}(t) \mathrm{d} t=\frac{k}{2^{k / 2} a^{2+1 / k} \Gamma(1+k / 2)} \int_{x}^{\infty} t^{2 k} \exp \left(-\frac{t^{2 k}}{2 a^{2}}\right) \mathrm{d} t \\
& =\frac{x}{2^{k / 2} a^{1 / k} \Gamma(1+k / 2)} \exp \left(-\frac{x^{2 k}}{2 a^{2}}\right)+\frac{1}{2^{k / 2} a^{1 / k} \Gamma(1+k / 2)} \int_{x}^{\infty} \exp \left(-\frac{t^{2 k}}{2 a^{2}}\right) \mathrm{d} t \\
& <\frac{1}{2^{k / 2} a^{1 / k} \Gamma(1+k / 2)} \exp \left(-\frac{x^{2 k}}{2 a^{2}}\right)\left(x+\left(\frac{a^{2}}{k} x^{2 k-1}-\frac{1}{x}\right)^{-1}\right) \\
& =g_{k}(x) \frac{a^{2}}{k} x^{1-2 k}\left(1+\left(\frac{a^{2}}{k} x^{2 k}-1\right)^{-1}\right) .
\end{aligned}
$$

The inequality above is derived by utilizing Lemma 2.1. Thus,

$$
\frac{G_{k}(-x)}{g_{k}(x)}<\frac{a^{2}}{k} x^{1-2 k}\left(1+\left(\frac{a^{2}}{k} x^{2 k}-1\right)^{-1}\right) .
$$

By using (2.1), we have

$$
\frac{G_{k}(-x)}{g_{k}(x)}>\frac{a^{2}}{k} x^{1-2 k}
$$

The result follows.

Theorem 2.1. For fixed $k>0$, we have

$$
\frac{G_{k}(-x)}{g_{k}(x)} \sim \frac{a^{2}}{k} x^{1-2 k}, \text { as } x \rightarrow \infty .
$$

Proof. For $0<k<\frac{1}{2}, g_{k}(x) x^{1-2 k} \rightarrow 0$, as $x \rightarrow \infty$, so we apply L'Hospital's rule to show that

$$
\lim _{x \rightarrow \infty} \frac{G_{k}(-x)}{x^{1-2 k} g_{k}(x)}=\lim _{x \rightarrow \infty} \frac{-g_{k}(x)}{(1-2 k) x^{-2 k} g_{k}(x)+g_{k}(x)\left(2 k x^{-2 k}-k a^{-2}\right)}=\frac{a^{2}}{k} .
$$

For $k=\frac{1}{2}$, easily check that $\left(1-G_{1 / 2}(x)\right) / g_{1 / 2}(x) \rightarrow 2 a^{2}$, as $x \rightarrow \infty$. For $k>\frac{1}{2}$, applying the inequality of Lemma 2.2, we can derive the desired result. The proof is complete. 
Remark 2.1. For $k>\frac{1}{2}$, the result of Theorem 2.1 can be applied to show that $G_{k} \in D(\Lambda)$, i.e., there exist normalizing constants $a_{n}>0$ and $b_{n} \in R$ such that $G_{k}\left(a_{n} x+b_{n}\right) \rightarrow \Lambda(x)=$ $\exp (-\exp (-x))$, as $n \rightarrow \infty$.

Since

$$
\frac{(d / d x) g_{k}(x)}{g_{k}(x)}=\frac{2 k}{x}-\frac{k x^{2 k-1}}{a^{2}},
$$

we have by Theorem 2.1 that

$$
\frac{G_{k}(-x)}{g_{k}(x)} \frac{(d / d x) g_{k}(x)}{g_{k}(x)} \rightarrow-1
$$

as $x \rightarrow \infty$, thus, by Proposition 1.18 in Resnick (1987) that $G_{k} \in D(\Lambda)$. We will discuss the choice of the constants $a_{n}$ and $b_{n}$ by Theorem 4.1.

Remark 2.2. If $k=1$, the above Theorem 2.1 yields the Mills-type ratio of MD, i.e.,

$$
\frac{G_{1}(-x)}{g_{1}(x)} \sim \frac{a^{2}}{x} \text {, as } x \rightarrow \infty \text {. }
$$

\section{Tail property of GMD}

Finner et al. (2008) considered the asymptotic behavior of the ratio of the Student's $t$ and normal distributions when $u=u(x)$ satisfies

$$
\lim _{x \rightarrow \infty} \frac{x^{4}}{u}=\beta \in[0, \infty),
$$

where $u$ is the degrees of freedom of the Student's $t$ distribution. The main motivation is to consider the false discovery rate in multiple testing problems with large numbers of hypotheses and extremely small critical values of the smallest ordered $p$ values, in detail, see Finner et al. (2007).

In this Section, we extend Finner et al. (2008)'s results to the GMD, that is, characterize asymptotic behavior of the pdfs and the ratio of the tails of the GMD and the MD.

Next we will consider the asymptotic behavior as $k \rightarrow 1$ in Theorem 3.1 while will consider the asymptotic behavior as $x \rightarrow \infty$ in Theorem 3.2.

Theorem 3.1. Let $x=x(k)$ be such that $k-1 \sim \gamma /\left\{2 x^{2} \log |x|\right\}$ as $k \rightarrow 1$ for $\gamma \in \mathbb{R}$. We have

$$
\lim _{k \rightarrow 1} \frac{g_{1}(x)}{g_{k}(x)}=\exp \left(\frac{\gamma}{2 a^{2}}\right)
$$

and

$$
\lim _{k \rightarrow 1} \frac{G_{1}(-x)}{G_{k}(-x)}=\exp \left(\frac{\gamma}{2 a^{2}}\right)
$$

Proof. Note that $\left\{2^{k / 2} a^{2+1 / k} \Gamma(1+k / 2)\right\} /\left\{k \sqrt{\pi / 2} a^{3}\right\} \rightarrow 1, k \rightarrow 1$, so, easily check (3.2). $x^{2 k-1} \rightarrow x$ as $k \rightarrow 1$ can be deduced by the condition of the theorem. Therefore, (3.3) follows by writing

$$
G_{1}(-x) / G_{k}(-x)=\left\{G_{1}(-x) / g_{1}(x)\right\}\left\{g_{1}(x) / g_{k}(x)\right\}\left\{g_{k}(x) / G_{k}(-x)\right\}
$$

and applying (3.2) and Theorem 2.1. 
Theorem 3.2. For fixed $k$, we have

$$
\frac{g_{1}(x)}{g_{k}\left(x^{1 / k}\right)}=\frac{2^{(1+k) / 2} a^{1 / k-1} \Gamma(1+k / 2)}{\sqrt{\pi} k}
$$

and

$$
\lim _{x \rightarrow \infty} \frac{G_{1}(-x)}{x^{1-1 / k} G_{k}\left(-x^{1 / k}\right)}=\frac{2^{(1+k) / 2} a^{1 / k-1} \Gamma(1+k / 2)}{\sqrt{\pi}} .
$$

Proof. It is easy to check (3.4) by elementary calculation. Combining with (2.3) and Theorem 2.1, we have

$$
\lim _{x \rightarrow \infty} \frac{G_{1}(-x)}{x^{1-1 / k} G_{k}\left(-x^{1 / k}\right)}=\lim _{x \rightarrow \infty} \frac{k g_{1}(x)}{g_{k}\left(x^{1 / k}\right)}=\frac{2^{(1+k) / 2} a^{1 / k-1} \Gamma(1+k / 2)}{\sqrt{\pi}},
$$

so the result follows.

Utilizing Theorem 3.2 is to obtain the explicit representation of $G_{k}(-x)$ given by Corollary 3.1 .

Corollary 3.1. Under the condition of Theorem 3.2, we have

$$
G_{k}(-x)=c(x) \exp \left(-\int_{1}^{x} \frac{g(t)}{f(t)} \mathrm{dt}\right)
$$

for $x$ sufficiently large, where

and

$$
\begin{gathered}
c(x)=\frac{\exp \left(-1 /\left(2 a^{2}\right)\right)}{2^{k / 2} a^{1 / k} \Gamma(1+k / 2)}\left(1+\theta_{3}(x)\right) \\
f(t)=\frac{a^{2}}{k} t^{1-2 k}
\end{gathered}
$$

$$
g(t)=1-\frac{a^{2}}{k} t^{-2 k}
$$

Proof. By Theorem 3.2, for large enough $x$, we have

$$
G_{k}\left(-x^{1 / k}\right)=\frac{\sqrt{\pi}}{2^{(1+k) / 2} a^{1 / k-1} \Gamma(1+k / 2)} x^{1 / k-1} G_{1}(-x)\left(1+\theta_{1}(x)\right)
$$

where $\theta_{1}(x) \rightarrow 0$ as $x \rightarrow \infty$. Setting $z=x^{1 / k}$ and using (2.3) from the result of Lemma 3.3, we can rewrite (3.6) as

$$
\begin{aligned}
G_{k}(-z) & =\frac{\sqrt{\pi}}{2^{(1+k) / 2} a^{1 / k-1} \Gamma(1+k / 2)} z^{1-k} G_{1}\left(-z^{k}\right)\left(1+\theta_{2}(z)\right) \\
& =\frac{1}{2^{k / 2} a^{1 / k} \Gamma(1+k / 2)} z \exp \left(-\frac{z^{2 k}}{2 a^{2}}\right)\left(1+\theta_{3}(z)\right) \\
& =\frac{1}{2^{k / 2} a^{1 / k} \Gamma(1+k / 2)} \exp \left(\log z-\frac{z^{2 k}}{2 a^{2}}\right)\left(1+\theta_{3}(z)\right) \\
& =\frac{\exp \left(-\frac{1}{2 a^{2}}\right)}{2^{k / 2} a^{1 / k} \Gamma(1+k / 2)}\left(1+\theta_{3}(z)\right) \exp \left(-\int_{1}^{z} \frac{1-a^{2} t^{-2 k} / k}{a^{2} t^{1-2 k} / k} \mathrm{dt}\right)
\end{aligned}
$$

where $\theta_{2}(z) \rightarrow 0$ and $\theta_{3}(z)=(1+o(1))\left(1+\theta_{2}(z)\right)-1 \rightarrow 0$, as $z \rightarrow \infty$. We derive the desired result.

Remark 3.1. As $\lim _{t \rightarrow \infty} g(t)=1, f(t)>0$ on $[1,+\infty)$ and $\lim _{t \rightarrow \infty} f^{\prime}(t)=0$ in Corollary 3.1, using Corollary 1.7 in Resnick (1987) and the result of Corollary 3.1, we have $G_{k} \in D(\Lambda)$. 


\section{Limiting Distribution of the Maxima}

The following work is to find the suitable norming constants which make sure the distribution of the maxima tends to $\exp (-\exp (-x))$.

Theorem 4.1. Let $\left\{X_{n}, n=1,2, \cdots\right\}$ be independent and identically distributed random variables with common GMD $G_{k}(x), k>0$. Let $M_{n}=\max \left(X_{i}, 1 \leq i \leq n\right)$ denote the partial maximum. We have

$$
\lim _{n \rightarrow \infty} P\left(M_{n} \leq a_{n} x+b_{n}\right)=\exp (-\exp (-x)),
$$

where

$$
\begin{gathered}
a_{n}=\frac{a^{1 / k}}{k(2 \log n)^{1-1 /(2 k)}}, \\
b_{n}=a^{1 / k}(2 \log n)^{1 /(2 k)}+\frac{a^{1 / k}\left[\log \log n+\left(1-k^{2}\right) \log 2-2 k \log \Gamma(1+k / 2)\right]}{2 k^{2}(\log n)^{1-1 /(2 k)}} .
\end{gathered}
$$

Proof. Because of the monotonicity and continuity of $G_{k}(x)$ on $(0, \infty)$, there must exist $u_{n}=u_{n}(x)$ which induces $n\left(1-G_{k}\left(u_{n}\right)\right)=\exp (-x)$. Hence, combining with $(2.2)$, we have

$$
\frac{n}{2^{k / 2} a^{1 / k} \Gamma(1+k / 2)} u_{n} \exp \left(-\frac{u_{n}^{2 k}}{2 a^{2}}+x\right) \rightarrow 1 \text {, as } n \rightarrow \infty \text {. }
$$

Thus we have

$$
\begin{array}{r}
\log n-\frac{k}{2} \log 2-\frac{1}{k} \log a-\log \Gamma\left(1+\frac{k}{2}\right)+\log u_{n} \\
-\frac{u_{n}^{2 k}}{2 a^{2}}+x \rightarrow 0, \text { as } n \rightarrow \infty .
\end{array}
$$

(4.1) implies

$$
\frac{u_{n}^{2 k}}{2 a^{2} \log n} \rightarrow 1
$$

Taking logarithms, we have

$$
\log u_{n}=\frac{1}{2 k}(\log \log n+\log 2+2 \log a)+o(1) .
$$

Putting (4.2) in (4.1) by some simple calculation, we have

$$
\begin{aligned}
u_{n} & =2^{\frac{1}{2 k}} a^{\frac{1}{k}}\left(\log n+\frac{1}{2 k} \log \log n+\frac{1}{2}\left(\frac{1}{k}-k\right)-\log \Gamma\left(1+\frac{k}{2}\right)+x+o(1)\right)^{\frac{1}{2 k}} \\
& =a^{\frac{1}{k}}(2 \log n)^{\frac{1}{2 k}}+\frac{a^{\frac{1}{k}}}{2 k^{2}(2 \log n)^{1-\frac{1}{2 k}}}\left(\log \log n+\left(1-k^{2}\right) \log 2-2 k \log \Gamma\left(1+\frac{k}{2}\right)\right) \\
& +\frac{a^{\frac{1}{k}}}{k(2 \log n)^{1-\frac{1}{2 k}}} x+o\left((\log n)^{\frac{1}{2 k}-1}\right) \\
& =b_{n}+a_{n} x+o\left((\log n)^{\frac{1}{2 k}-1}\right)
\end{aligned}
$$

By Theorem 1.2.3 in Leadbetter et al. (1983), the proof is completed. 
Remark 4.1. As aforementioned, we get the classical Maxwell pdf when $k=1$ in (1.1). Theorem 4.1 shows that the limiting distribution of the maximum from the classical Maxwell is Gumbel extreme value distribution with the normalizing constants

$$
a_{n}=\frac{a}{(2 \log n)^{1 / 2}}
$$

and

$$
b_{n}=a(2 \log n)^{1 / 2}+\frac{a \log (2 \log n)+a \log (2 / \pi)}{2(2 \log n)^{1 / 2}} .
$$

\section{Acknowledgements}

The authors would like to thank the referees for their careful reading of the manuscript and many constructive comments which greatly improved the article. Meanwhile, the authors would like to thank the Editor-in-Chief for his careful work on this paper.

\section{References}

[1] Arbab, A. I., Satti, Z. A. (2009). On the Generalized Maxwell Equations and Their Prediction of Electroscalar Wave. Progress in Physics, 2, 8-13.

[2] Farid, A., Vincent, L., Jan, S. (2005). IEEE Transactions on Automatic Control, 50, 1883-1887.

[3] Finner, H., Dickhaus, T., Roters, M. (2008). Asymptotic tail properties of Students t-distribution. Communications in Statistics-Theory and Methods, 37,175-179.

[4] Finner, H., Dickhaus, T., Roters, M. (2007). Dependency and false discovery rate: asymptotics. the Annals of Statistics, 35,1432-1455.

[5] Leadbetter, M. R., Lindgren, G. and Rootzén, H. (1983). Extremes and Related Properties of Random Sequences and Processes. New York: Springer-Verlag.

[6] Lin, F., Peng, Z. (2010). Tail behavior and extremes of short-tailed symmetric distribution. Communications in Statistics-Theory and Methods, 39, 2811-2817.

[7] Lin, F., Jiang, Y. (2012) A General Version of the Short-Tailed Symmetric Distribution. Communications in Statistics-Theory and Methods, 41, 2088-2095.

[8] Liu, B. and Fu, Y. (2013). The point-wise rate of convergence of Maxwell distribution. Journal of Southwest University (Natural Science Edition), 35, 80-83.

[9] Liu, C. and Liu, B. (2013). Convergence rate of extremes from Maxwell sample. Journal of Inequalities and Applications 2013:477. Available on-line at http://www.journalofinequalitiesandapplications.com/content/2013/1/477.

[10] Mills, J. P. (1926). Table of the ratio: Area to bounding ordinate, for any portion of normal curve. Biometrika, 18, 359-400.

[11] Monsia, M. D. (2011). A Simplified Nonlinear Generalized Maxwell Model for Predicting the Time Dependent Behavior of Viscoelastic Materials. World Journal of Mechanics, 1, 158-167.

[12] Peng, Z., Tong, B., Nadarajah, S. (2009). Tail behavior of the general error distribution. Communications in Statistics-Theory and Methods, 38, 1884-1892.

[13] Resnick, S. I. (1987). Extreme value, Regular Variation, and Point Processes. New York: Springer. 
[14] Schiessel, H., Metzler, R., Blumen, A., Nonnenmacher, T. F. (1995). Generalized viscoelastic model: their fractional equations with solutions. Journal of Physics A: Mathematical and General, 28, 65676584 .

[15] Soms, A. P. (1983). Bounds for the t-tail area. Communications in Statistics-Theory and Methods, 12, 559-568.

[16] Soms, A. P. (1984). Note on an extension of rational bounds for the $t$-tail area to arbitrary degrees of freedom. Communications in Statistics-Theory and Methods, 13, 887-891.

[17] Vodă, V. G. (2009). A modified Weibull hazard rate as generator of a generalized Maxwell distribution. Mathematical Reports (Bucuresti), 11, 171-179.

[18] Zhang, W., Guo, X., Kassab, G. S. (2008). A Generalized Maxwell Model for Creep Behavior of Artery Opening Angle. Journal of Biomechanical Engineering, 130, 1-16. 